\title{
Expression of microRNA-26b, an obesity-related microRNA, is regulated by free fatty acids, glucose, dexamethasone and growth hormone in human adipocytes
}

\author{
GUANGFENG XU ${ }^{1,2,3 *}$, CHUNMEI SHI ${ }^{2,3 *}$, CHENBO JI $^{2}$, GUIXIAN SONG ${ }^{4}$, LING CHEN $^{3}$, \\ LEI YANG $^{3}$, YAPING ZHAO ${ }^{1}$ and XIRONG GUO ${ }^{2,3}$
}

\author{
${ }^{1}$ Department of Laboratory Medicine, The 82nd Hospital of The People's Liberation Army, Huaian, Jiangsu 223001; \\ ${ }^{2}$ Department of Children Health Care, Nanjing Maternity and Child Health Care Hospital Affiliated to \\ Nanjing Medical University; ${ }^{3}$ Institute of Pediatrics, Nanjing Medical University; ${ }^{4}$ Department of Cardiology, \\ The First Affiliated Hospital of Nanjing Medical University, Nanjing, Jiangsu 210029, P.R. China
}

Received August 30, 2013; Accepted April 3, 2014

DOI: $10.3892 / \mathrm{mmr} .2014 .2204$

\begin{abstract}
MicroRNAs (miRNAs) are short non-coding RNAs that are involved in numerous biological processes, including obesity and insulin resistance. miR-26b is an obesity-related intronic miRNA located in the intron of the carboxy-terminal domain, RNA polymerase II, polypeptide A, small phosphatase 1 gene. miR-26b is abundantly expressed in mice and mature human adipocytes, and is associated with the expression of adipokines. In the present study, the effects of energy-source materials and hormones associated with obesity, on miR-26b expression were investigated. It was demonstrated that free fatty acids (FFAs), glucose, glucocorticoids and growth hormone (GH) downregulate the expression of miR-26b in human adipocytes. The results indicate that the expression of miR-26b is affected by a variety of factors that are correlated with obesity and insulin sensitivity. Therefore, miR-26b may be an important mediator in the development of obesity-associated insulin resistance.
\end{abstract}

Correspondence to: Dr Yaping Zhao, Department of Laboratory Medicine, The 82nd Hospital of The People's Liberation Army, 100 Jiankang East Road, Huaian, Jiangsu 223001, P.R. China E-mail: 549744462@qq.com

Dr Xirong Guo, Department of Children Health Care, Nanjing Maternity and Child Health Care Hospital Affiliated to Nanjing Medical University, 123 Tianfei Road, Nanjing, Jiangsu 210029, P.R. China

E-mail:xrguo@njmu.edu.cn

*Contributed equally

Key words: microRNA-26b, adipocyte, free fatty acids, glucose, growth hormone, dexamethasone

\section{Introduction}

In recent years, obesity has become a global public health problem, associated with a number of complications and increasing prevalence among children and adolescents $(1,2)$. Obesity is characterized as an excessive accumulation of adipose tissue due to severe energy imbalance (3) and it is associated with dyslipidemia, type-2 diabetes mellitus, cardiovascular disease, cancer and obstructive sleep apnea $(4,5)$. As energy-source materials, free fatty acids (FFAs) and glucose are important in obesity and obesity-related insulin sensitivity. Furthermore, studies have indicated that glucocorticoids and growth hormone $(\mathrm{GH})$ are also closely associated with obesity and insulin resistance $(6,7)$. However, the molecular mechanisms underlying the effects of FFAs, glucose, glucocorticoids and GH in obesity and insulin sensitivity, have not yet been fully clarified.

MicroRNAs (miRNAs) are endogenous 22 nucleotide RNAs that have important regulatory roles in animal and plant physiology, by targeting mRNAs for cleavage or translational repression (8). Emerging evidence has suggested that a number of miRNAs are important in the regulation of adipocyte differentiation, including the miR-143 (9) and miR-1792 clusters (10). miR-26b is an intronic miRNA encoded in the carboxy-terminal domain, RNA polymerase II, polypeptide A, small phosphatase 1 (CTDSPI) gene that is important in a variety of different diseases, including lung carcinoma, breast cancer and cardiac hypertrophy (11-13). However, there have been few studies investigating the role of miR-26b in obesity and insulin sensitivity. Previously, Klöting et al (14) indicated that miR-26b is closely correlated with the number of infiltrating macrophages in abdominal subcutaneous (SC) adipose tissue. In addition, it appears that miR-26b is upregulated during adipogenesis in mice and humans $(15,16)$. In our preliminary study, it was identified that miR-26b is increased during adipogenesis of human multipotent adipose-derived stem cells and preadipocytes screened from miRNA microarray experiments (unpublished data). Therefore, it is likely that miR-26b is associated with 
obesity and obesity-related insulin resistance. Investigation into the correlation between miR-26b and energy-source materials or hormones that are associated with obesity and obesity-related insulin resistance, may be critical for enhancing our understanding of the mechanisms of obesity development and may aid in the development of strategies that promote obesity prevention and control.

The aim of the present study was to examine the effects of energy-source materials (FFAs and glucose) or hormones (glucocorticoids and GH) associated with obesity and obesity-related insulin resistance on miR-26b expression in human adipocytes, and to clarify the role of miR-26b in regulating obesity development and insulin resistance.

\section{Materials and methods}

Cell culture and differentiation. Human preadipocytes (ScienCell Research Laboratories, San Diego, CA, USA) were maintained in preadipocyte medium (PAM; ScienCell Research Laboratories) supplemented with 5\% fetal bovine serum (FBS), $1 \%$ preadipocyte growth supplement and $1 \%$ penicillin/streptomycin solution $(\mathrm{P} / \mathrm{S})$ at $37^{\circ} \mathrm{C}$ in a humidified atmosphere of $5 \% \mathrm{CO}_{2}$. To induce differentiation, confluent human preadipocytes (day 0) were cultured in serum-free PAM containing $50 \mathrm{nM}$ insulin, $100 \mathrm{nM}$ dexamethasone (DEX), $0.5 \mathrm{mM} 3$-isobutyl-1-methylxanthine and $100 \mu \mathrm{M}$ rosiglitazone. The medium was changed every two days for the first four days. Following this, the medium was replaced with serum-free PAM containing $50 \mathrm{nM}$ insulin, which was changed every two days until the accumulation of lipid droplets was observed (day 15). Subsequently, when $>75 \%$ of the cells exhibited the morphological and biochemical properties of adipocytes, the cells were selected to be used in the experiments.

Oil red $O$ staining. For oil red $\mathrm{O}$ staining, cell culture plates were retrieved and the medium was removed. Adipocytes were washed three times with phosphate-buffered saline (PBS) and fixed with $4 \%$ formalin in phosphate buffer for $30 \mathrm{~min}$ at room temperature. Following fixation, cells were washed twice with PBS and stained with $0.6 \%$ (w/v) filtered oil red $\mathrm{O}$ solution (60\% isopropanol, $40 \%$ water) for $30 \mathrm{~min}$ at room temperature. Following this, cells were washed with tap water to remove unbound dye, visualized by light microscopy (Olympus, Tokyo, Japan) and photographed (Olympus, Tokyo, Japan).

Interference of adipocytes with FFAs, glucose, glucocorticoids and GH. Following overnight incubation in serum-free PAM, human adipocytes were treated with different adipokines, respectively, including $1 \mathrm{mmol} / 1$ FFA cocktail (lauric, myristic, linoleic, oleic and arachidonic acids), glucose (5 or $25 \mathrm{mmol} / \mathrm{l}$ ), $1 \mathrm{mmol} / 1 \mathrm{DEX}$ and $100 \mathrm{nmol} / \mathrm{l} \mathrm{GH}$ (Sigma, St. Louis, MO, USA) for different durations of time (4, 8 and 24 or 48 h). Adipocytes were collected at each time-point and prepared for further investigation.

RNA isolation and quantitative (q)PCR. Total RNA was extracted from adipocytes using TRIzol reagent (Invitrogen Life Technologies, Carlsbad, CA, USA) and quantified spec- trophotometrically (NanoDrop Technology, Rockland, DE, USA) at $260 \mathrm{~nm}$. miRNA quantification was performed via TaqMan miRNA analysis of miR-26b. Generation of cDNA was performed using the TaqMan microRNA reverse transcription kit (Applied Biosystems, Foster City, CA, USA) in $15 \mu \mathrm{l}$ reverse transcriptase reactions containing $70 \mathrm{ng}$ total RNA, $50 \mathrm{nM}$ stem-loop RT primer, 1X RT buffer, $0.25 \mathrm{mM}$ each dNTP, $3.33 \mathrm{U} / \mathrm{ml}$ Multi-Scribe RT and $0.25 \mathrm{U} / \mathrm{ml}$ RNase inhibitor. Reaction conditions were as previously described (16). For qPCR, $1.33 \mu \mathrm{l}$ (dilution, 1:15) cDNA, $0.2 \mathrm{mM}$ TaqMan probe, $1.5 \mathrm{mM}$ forward primer, $0.7 \mathrm{mM}$ reverse primer and TaqMan Universal PCR Master mix (Applied Biosystems) were included in $20 \mu \mathrm{l}$ reactions. The RT primer, PCR primer and Taq Man probe for miR-26b were purchased from Applied Biosystems. Reaction conditions were as previously described (16). The qPCR results were analyzed and expressed relative to the miRNA expression of CT (threshold cycle) value. The RT primer, PCR primer and TaqMan probe for miR-26b were purchased from (Applied Biosystems). U6 small nucleolar RNA (snRU6) and miR-103 were used as references to obtain the relative fold-change in expression in target samples, using the comparative CT method.

Statistical analysis. The data are presented as the mean \pm standard error of the mean. Statistical analysis was performed using one-way analysis of variance. $\mathrm{P}<0.05$ was considered to indicate a statistically significant difference.

\section{Results}

Assessment of lipid accumulation. Through oil red $\mathrm{O}$ staining, it was observed that $>75 \%$ of the human preadipocytes differentiated into mature adipocytes, which were large, round and filled with fat droplets. These data suggested that the majority of the adipocytes had matured (Fig. 1).

Effects of FFAs on miR-26b expression in human adipocytes. The effects of $1 \mathrm{mmol} / 1$ FFAs on miR-26b expression in cultured human adipocytes were analyzed using qPCR (TaqMan probe method). Differentiation of human preadipocytes was induced and adipocyte cultures were prepared for use in experiments as described in the Materials and methods. Adipocytes were cultured in the presence of $1 \mathrm{mmol} / \mathrm{l} \mathrm{FFAs}$. Expression of miR-26b was significantly downregulated in a time-dependent manner from $4 \mathrm{~h}$ following the initiation of stimulation by FFAs. This effect was maintained for up to 24 h (Fig. 2).

Effects of glucose on miR-26b expression in human adipocytes. To assess the effects of glucose on miR-26b expression, the miR-26b expression in human adipocytes treated with 5 or $25 \mathrm{mmol} / \mathrm{l}$ glucose for different durations (4, 8, 24 and $48 \mathrm{~h}$ ) was examined on the 15 th day of differentiation. It was identified that glucose concentrations of 5 or $25 \mathrm{mmol} / \mathrm{l}$ led to a time-dependent decrease in the miR-26b expression and this effect was more potently induced by $25 \mathrm{mmol} / 1$ glucose. As shown in Fig. 3, miR-26b expression significantly decreased after only $12 \mathrm{~h}$ of exposure. Following this, the levels of miR-26b expression remained relatively low at 24-48 h, at 


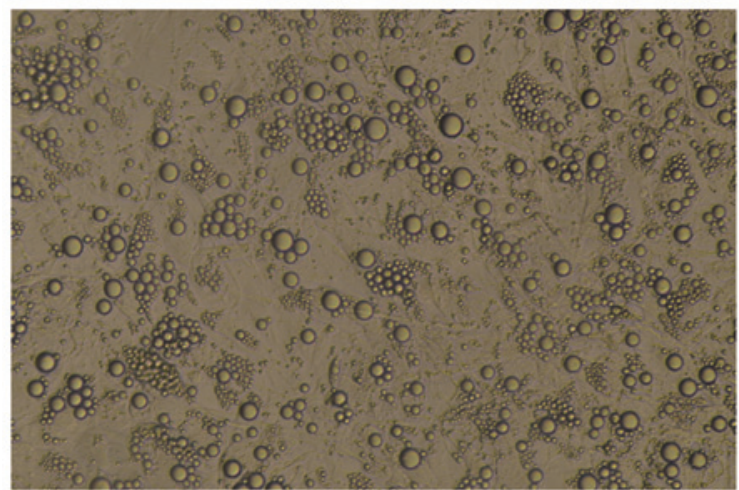

Day 15 (unstained cell)

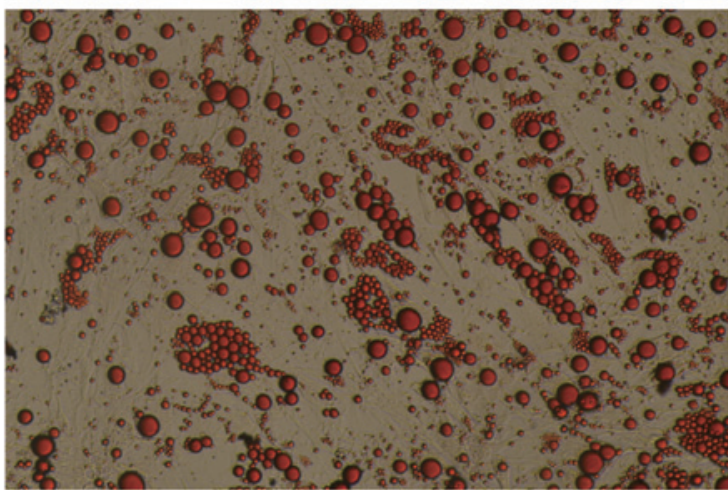

Day 15 (stained with oil red O)

Figure 1. On day 15 of differentiation, the cells were fixed and stained with oil Red O to visualize lipids as described in the Materials and methods. On day 15 , the morphological changes associated with cell differentiation were captured following oil Red O staining (magnification, $\mathrm{x} 200$ ).
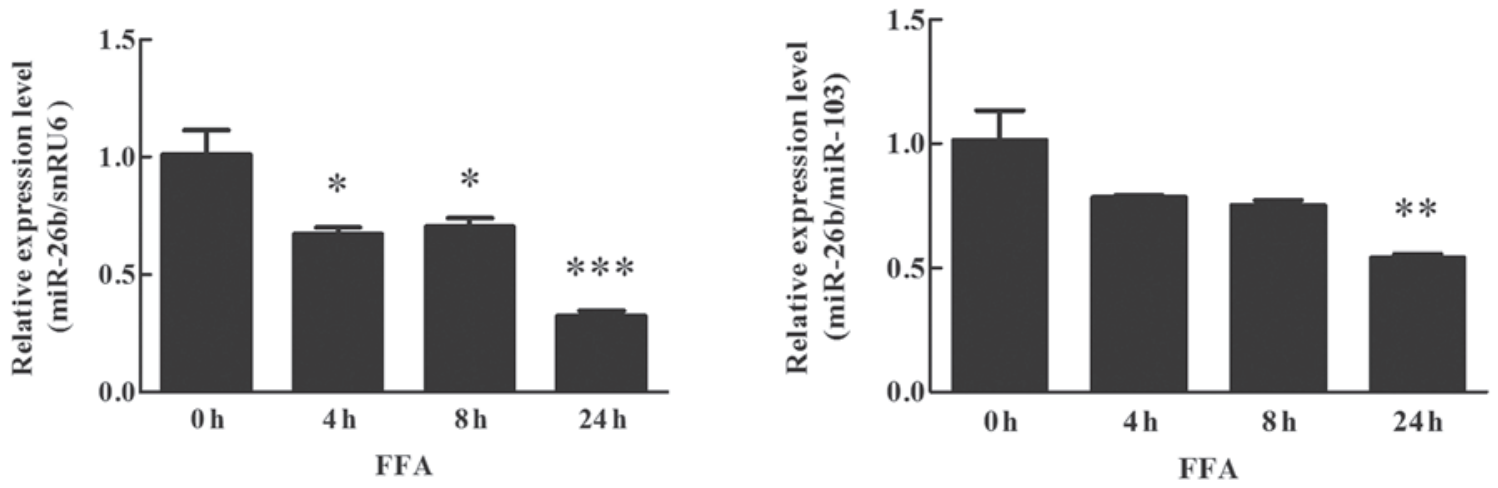

Figure 2. Effects of FFAs on miR-26b expression in human adipocytes. Differentiated human adipocytes were treated with 1 mmol/1 FFAs for the indicated periods (up to $24 \mathrm{~h}$ ). miR-26b expression was analyzed using quantitative polymerase chain reaction and normalized to the snRU6 and miR-103 levels. Results are presented as the mean \pm standard errror of the mean of three experiments. ${ }^{*} \mathrm{P}<0.05,{ }^{* * *} \mathrm{P}<0.01$ and ${ }^{* * *} \mathrm{P}<0.001$, compared with the untreated cells $(0 \mathrm{~h})$. miR, microRNA; FFAs, free fatty acids; snRU6, U6 small nucleolar RNA.
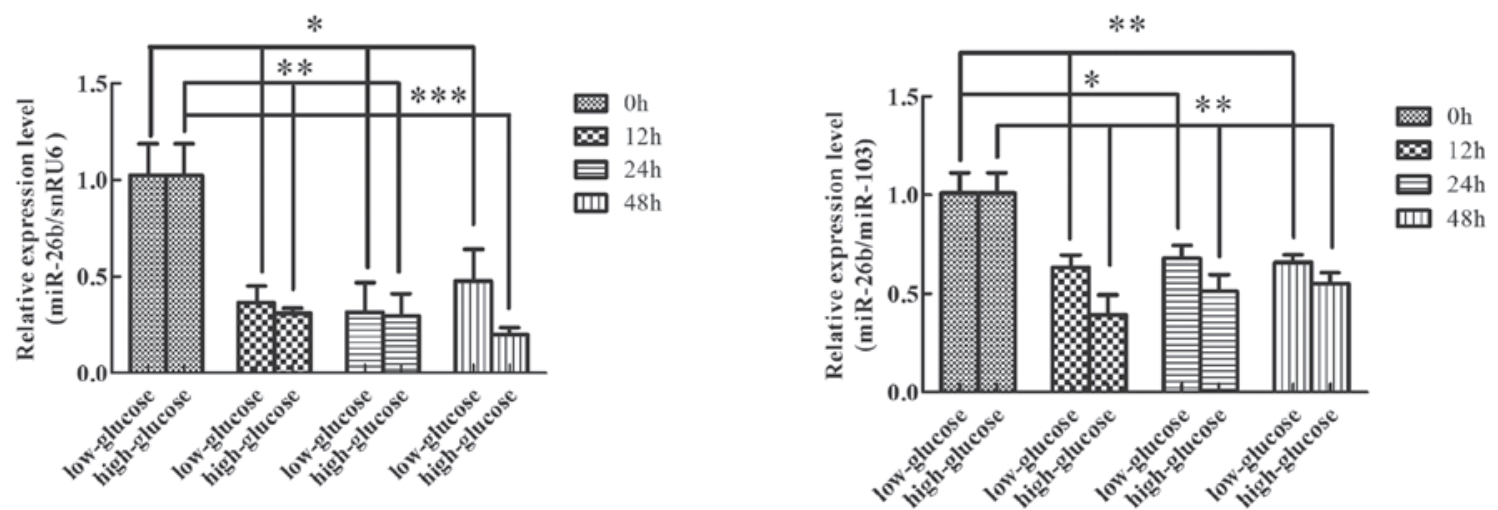

Figure 3. Effects of glucose on miR-26b expression in human adipocytes. Matured human adipocytes were treated with low $(5 \mathrm{mmol} / \mathrm{l}) \mathrm{or}$ high $(25 \mathrm{mmol} / \mathrm{l})$ glucose for the indicated periods (up to $48 \mathrm{~h}$ ). Expression of miR-26b was quantified by quantitative polymerase chain reaction and normalized to the snRU6 and miR-103 levels. Values are represented as the mean \pm standard error of the mean from three independent experiments $(\mathrm{n}=3)$. ${ }^{*} \mathrm{P}<0.05,{ }^{* *} \mathrm{P}<0.01$ and **** $\mathrm{P}<0.001$ vs. the untreated cells $(0 \mathrm{~h})$. miR, microRNA; snRU6, U6 small nucleolar RNA.

which time the expression of miR-26b was $\sim 3$-fold lower than that in the control $(\mathrm{P}<0.05)$.

Effects of DEX on miR-26b expression in human adipocytes. The effects of $1 \mathrm{mmol} / 1 \mathrm{DEX}$ on miR-26b expression in cultured human adipocytes were analyzed using qPCR (TaqMan probe method). Mature adipocytes were cultured in the presence of $1 \mathrm{mmol} / \mathrm{l} \mathrm{DEX}$. Expression of miR-26b was identified to be significantly altered by DEX stimulation. As demonstrated in Fig. 4, miR-26b expression markedly 

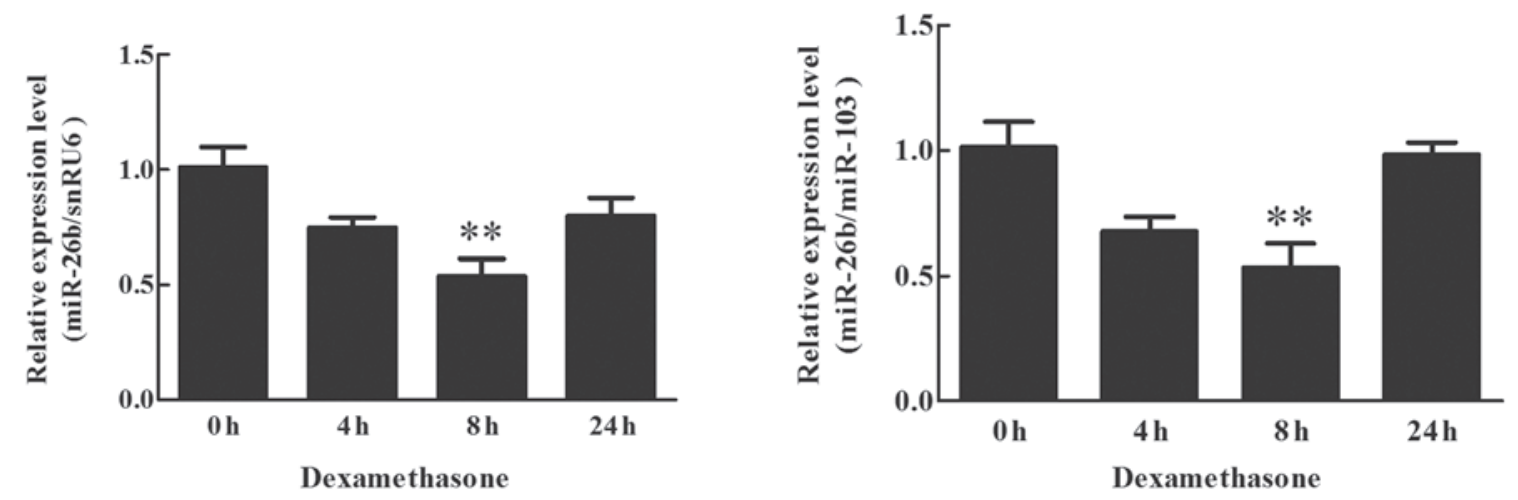

Figure 4. Effects of DEX on miR-26b expression in human adipocytes. The levels of miR-26b of differentiated human adipocytes were analyzed by quantitative polymerase chain reaction following treatment with $1 \mathrm{mmol} / \mathrm{l} \mathrm{DEX}$ for the indicated periods (up to $24 \mathrm{~h}$ ). Results are presented as the mean $\pm \mathrm{SEM}$ of three experiments. Basal levels were defined at $0 \mathrm{~h}$ (untreated cells). ${ }^{* *} \mathrm{P}<0.01$ vs. untreated cells $(0 \mathrm{~h})$. miR, microRNA; DEX, dexamethasone; snRU6, U6 small nucleolar RNA.
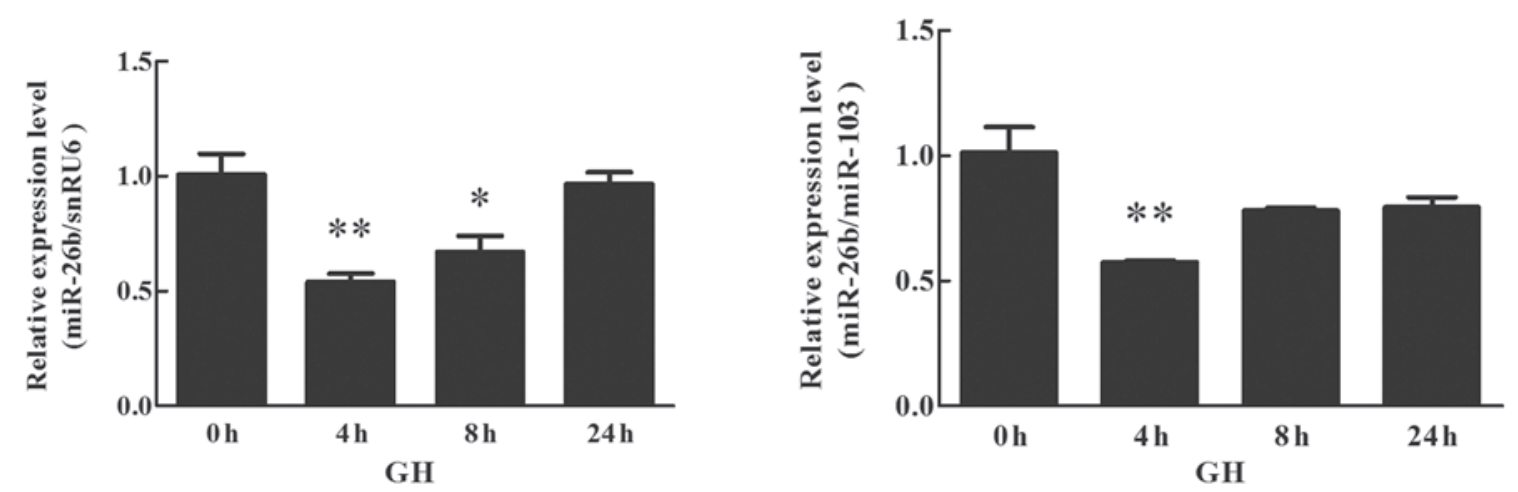

Figure 5. Effects of GH on miR-26b expression in human adipocytes. Differentiated human adipocytes were treated with $100 \mathrm{nmol} / 1 \mathrm{GH}$ for the indicated periods (up to $24 \mathrm{~h}$ ). miR-26b levels were analyzed by qPCR and normalized to the snRU6 and miR-103 levels. Values are presented as the mean \pm standard error of the mean of three experiments. ${ }^{*} \mathrm{P}<0.05$ and ${ }^{* *} \mathrm{P}<0.01$ vs. the miR-26b levels at $0 \mathrm{~h}$ (untreated cells). miR, microRNA; GH, growth hormone; qPCR, quantitative PCR; snRU6, U6 small nucleolar RNA.

decreased after $4-8 \mathrm{~h}$ of exposure. Following this, the levels of miR-26b expression were moderately increased. However, the expression of miR-26b remained decreased in comparison with the untreated cells.

Effects of GH on miR-26b expression in human adipocytes. The effects of $100 \mathrm{nmol} / \mathrm{l} \mathrm{GH}$ on miR-26b expression in cultured human adipocytes were analyzed using qPCR (TaqMan probe method). Differentiation of human preadipocytes was induced and adipocyte cultures were prepared for use in the experiments. The miR-26b expression in human adipocytes treated with $100 \mathrm{nmol} / \mathrm{l} \mathrm{GH}$ for different durations $(4,8$ and $24 \mathrm{~h})$ was detected. The expression of miR-26b was significantly downregulated in a time-dependent manner at $4 \mathrm{~h}$ following the initiation of GH stimulation. As demonstrated in Fig. 5, miR-26b expression markedly decreased after $4 \mathrm{~h}$ of exposure. Following this, the levels of miR-26b expression were marginally increased. However, the expression of miR-26b remained relatively lower than that in the untreated cells.

\section{Discussion}

Due to increased morbidity and mortality, obesity and obesity-associated diseases have become a major public health problem worldwide. Currently, the prevalence of obesity and obesity-associated diseases among children and adolescents, which is a vulnerable age for the development of obesity, is also increasing at an alarming rate (17). Although childhood and adolescent obesity is correlated with a number of additional problems, including hyperinsulinemia, poor glucose tolerance and a raised risk of type 2 diabetes, hypertension, sleep apnea, social exclusion and depression, the greatest health problems will emerge as the present childhood obesity epidemic progresses to the next generation of adults (18). Therefore, prevention of childhood and adolescent obesity is a priority. In particular, the population of adolescents who are already obese, urgently require strategies to prevent their progression into adulthood obesity.

Obesity is caused by an excessive accumulation of adipose tissue. Adipose tissue is composed of functional units termed adipocytes, is involved in the regulation of energy and glucose homeostasis, and appears to function as an endocrine organ $(19,20)$. Therefore, assessing its associations with energy-source materials and hormones is important for combating obesity and its complications. However, the molecular mechanisms underlying the effects of energy-source materials or hormones on obesity-related insulin sensitivity have not yet been fully clarified. 
miRNAs are a novel class of small, non-coding RNA molecules that function as negative gene regulators $(8,21)$. miRNA expression levels vary spatially and temporally in vivo and alter in response to internal and external signals. miRNA dysregulation is often associated with disease states, including obesity and insulin resistance. For example, Kloting et al (14) isolated miRNA from different fat depots of overweight and obese individuals in 2009 and Zhao et al (22) profiled 220 miRNAs in pancreatic islets, adipose tissue and liver isolated from diabetes-resistant (B6) and diabetes-susceptible (BTBR) mice. miR-26b is an intronic miRNA located in the intron of the CTDSP1 gene, which is able to regulate its host transcript (23). Emerging evidence has indicated that miR-26b is associated with the development of obesity. Several studies have demonstrated this, including in abdominal subcutaneous adipose tissue where miR-26b was closely correlated with the number of infiltrating macrophages (14) and was upregulated during adipogenesis in mice (15). In our previous study, it was also demonstrated that miR-26b was differentially expressed during differentiation of human preadipocytes and was affected by a number of adipokines, including tumor necrosis factor- $\alpha$ (TNF- $\alpha$ ), leptin and resistin (16). This evidence suggests that miR-26b is closely associated with the development of obesity. Thus, it was hypothesized that energy-source materials or hormones may participate in regulating the development of obesity via interacting with miR-26b.

Increased circulating concentrations of fatty acids and glucose are prominent features of type 2 diabetes and are also involved in the development of obesity and insulin resistance. FFA accumulation triggers several components of the insulin resistance syndrome and increases the risk of diabetes (24). Furthermore, plasma FFA concentrations are usually elevated in obese individuals (25). By contrast, obesity-induced glucose intolerance reflects a failure to mount one or more compensatory response through insulin-secreting $\beta$ cells to systemic insulin resistance (26). Therefore, FFAs and glucose are important in obesity and insulin resistance, however, the details of the mechanisms underlying their effects remain elusive. In the present study, it was observed that FFAs and glucose inhibit miR-26b expression in human adipocytes, respectively, and that the effect of high glucose levels on miR-26b was more potent, in comparison with low glucose levels. Due to the fact that elevated circulating concentrations of fatty acids and glucose are associated with insulin resistance, miR-26b may be involved in regulating the development of obesity and insulin resistance via increasing the insulin sensitivity of human adipocytes.

Glucocorticoids and GH have important roles in lipolysis and are able to increase plasma glucose levels. Numerous studies have indicated that high glucocorticoids in obese adipose tissue promotes the uptake and storage of fatty acids by increasing lipoprotein lipase levels (27), lipogenesis and lipid storage $(28,29)$. Furthermore, $\mathrm{GH}$ has a pronounced lipolytic effect, particularly in abdominal fat (30). In the present study, it was identified that DEX and GH decrease the expression of miR-26b in human adipocytes. However, DEX and GH have potent effects for only a relatively short time, and with time their actions become weaker. Therefore it was observed that DEX and GH participate in the development of obesity and insulin resistance via controlling miR-26b expression in human adipocytes. However, the time-dependent decrease of their effects suggests the involvement of other mechanisms.

In conclusion, the present study revealed that the expression of miR-26b is affected by FFAs, glucose, DEX and GH in human adipocytes. These obesity-associated factors are closely correlated with the development of obesity and insulin resistance, and it is therefore possible that miR-26b is involved in obesity-related insulin resistance. Thus, we hypothesize that miR-26b is involved in the progression and development of obesity and insulin resistance. To determine whether these effects occur independently further investigation is required.

\section{Acknowledgements}

This study was supported by grants from the National Key Basic Research Program of China (grant no. 2013CB530604), the National Natural Science Foundation of China (grant no. 81170797), the Natural Science Foundation of Jiangsu Province China (grant no. BK2011107), the Program for Innovative Research Teams of Jiangsu Province (grant no. LJ201108) and Nanjing Technological Development Program (grant no. 201104013).

\section{References}

1. Basham P, Luik J, Jeffery RW, et al: Is the obesity epidemic exaggerated? Yes. BMJ 336: 244, 2008

2. Haslam DW and James WP: Obesity. Lancet 366: 1197-1209, 2005.

3. Gregoire FM, Smas CM and Sul HS: Understanding adipocyte differentiation. Physiol Rev 78: 783-809, 1998.

4. Kassi E, Pervanidou P, Kaltsas G and Chrousos G: Metabolic syndrome: definitions and controversies. BMC Med 9: 48, 2011.

5. Lubrano C, Saponara M, Barbaro G, et al: Relationships between body fat distribution, epicardial fat and obstructive sleep apnea in obese patients with and without metabolic syndrome. PloS One 7: e47059, 2012.

6. Lee MJ and Fried SK: Glucocorticoids antagonize tumor necrosis factor- $\alpha$-stimulated lipolysis and resistance to the antilipolytic effect of insulin in human adipocytes. Am J Physiol Endocrinol Metab 303: E1126-E1133, 2012.

7. Morita J, Hakuno F, Hizuka N, Takahashi S and Takano K: Growth hormone $(\mathrm{GH})$ or insulin-like growth factor (IGF)-I represses 11beta-hydroxysteroid dehydrogenase type 1 (HSD1) mRNA expression in 3T3-L1 cells and its activity in their homogenates. Endocr J 56: 561-570, 2009.

8. Bartel DP: MicroRNAs: genomics, biogenesis, mechanism, and function. Cell 116: 281-297, 2004.

9. Esau C, Kang X, Peralta E, et al: MicroRNA-143 regulates adipocyte differentiation. J Biol Chem 279: 52361-52365, 2004.

10. Wang Q, Li YC, Wang J, et al: miR-17-92 cluster accelerates adipocyte differentiation by negatively regulating tumor-suppressor Rb2/p130. Proc Natl Acad Sci USA 105: 2889-2894, 2008.

11. Arora H, Qureshi R, Park AK and Park WY: Coordinated regulation of ATF2 by miR-26b in $\gamma$-irradiated lung cancer cells. PloS One 6: e23802, 2011.

12. Li J, Kong X, Zhang J, Luo Q, Li X and Fang L: MiRNA-26b inhibits proliferation by targeting PTGS2 in breast cancer. Cancer Cell Int 13: 7, 2013.

13. Han M, Yang Z, Sayed D, et al: GATA4 expression is primarily regulated via a miR-26b-dependent post-transcriptional mechanism during cardiac hypertrophy. Cardiovasc Res 93: 645-654, 2012.

14. Klöting N, Berthold S, Kovacs P, et al: MicroRNA expression in human omental and subcutaneous adipose tissue. PloS One 4: e4699, 2009.

15. Kajimoto K, Naraba $\mathrm{H}$ and Iwai N: MicroRNA and 3T3-L1 pre-adipocyte differentiation. RNA 12: 1626-1632, 2006.

16. Xu G, Ji C, Shi C, et al: Modulation of hsa-miR-26b levels following adipokine stimulation. Mol Biol Rep 40: 3577-3582, 2013. 
17. Ebbeling CB, Pawlak DB and Ludwig DS: Childhood obesity: public-health crisis, common sense cure. Lancet 360: 473-482, 2002.

18. Lobstein T, Baur L and Uauy R; IASO International Obesity TaskForce: Obesity in children and young people: a crisis in public health. Obes Rev 5 (Suppl 1): 4-104, 2004.

19. Rosen ED and Spiegelman BM: Adipocytes as regulators of energy balance and glucose homeostasis. Nature 444: 847-853, 2006.

20. Galic S, Oakhill JS and Steinberg GR: Adipose tissue as an endocrine organ. Mol Cell Endocrinol 316: 129-139, 2010.

21. Ambros V: The functions of animal microRNAs. Nature 431 350-355, 2004.

22. Zhao E, Keller MP, Rabaglia ME, et al: Obesity and genetics regulate microRNAs in islets, liver, and adipose of diabetic mice. Mamm Genome 20: 476-485, 2009.

23. Dill H, Linder B, Fehr A and Fischer U: Intronic miR-26b controls neuronal differentiation by repressing its host transcript, ctdsp2. Genes Dev 26: 25-30, 2012.

24. Bergman RN and Ader M: Free fatty acids and pathogenesis of type 2 diabetes mellitus. Trends Endocrinol Metab 11: 351-356, 2000.
25. Boden G: Role of fatty acids in the pathogenesis of insulin resistance and NIDDM. Diabetes 46: 3-10, 1997.

26. Kahn SE, Hull RL and Utzschneider KM: Mechanisms linking obesity to insulin resistance and type 2 diabetes. Nature 444: 840-846, 2006.

27. Fried SK, Russell CD, Grauso NL, et al: Lipoprotein lipase regulation by insulin and glucocorticoid in subcutaneous and omental adipose tissues of obese women and men. J Clin Invest 92: 2191-2198, 1993.

28. Lee MJ, Gong DW, Burkey BF and Fried SK: Pathways regulated by glucocorticoids in omental and subcutaneous human adipose tissues: a microarray study. Am J Physiol Endocrinol Metab 300: E571-E580, 2011.

29. Yu CY, Mayba O, Lee JV, et al: Genome-wide analysis of glucocorticoid receptor binding regions in adipocytes reveal gene network involved in triglyceride homeostasis. PloS One 5: e15188, 2010

30. Gravhølt CH, Schmitz O, Simonsen L, Bülow J, Christiansen JS and Møller N: Effects of a physiological GH pulse on interstitial glycerol in abdominal and femoral adipose tissue. Am J Physiol 277: E848-E854, 1999. 